\title{
Linking key intervention timings to rapid declining effective reproduction number to quantify lessons against COVID-19
}

\author{
Zhihang Peng ${ }^{1}$, Wenyu Song ${ }^{1}$, Zhongxing Ding ${ }^{1}$, Quanquan $\mathrm{Guan}^{2}, \mathrm{Xu} \mathrm{Yang}^{2}$, Qiaoqiao $\mathrm{Xu}^{2}, \mathrm{Xu} \mathrm{Wang}^{2}$, Yankai Xia $(\bowtie)^{2}$ \\ ${ }^{1}$ Department of Epidemiology and Biostatistics, School of Public Health, Nanjing Medical University, Nanjing 211166, China; ${ }^{2}$ Key \\ Laboratory of Modern Toxicology of Ministry of Education, School of Public Health, Nanjing Medical University, Nanjing 211166, China
}

(C) Higher Education Press 2020

\begin{abstract}
Coronavirus disease 2019 (COVID-19) is currently under a global pandemic trend. The efficiency of containment measures and epidemic tendency of typical countries should be assessed. In this study, the efficiency of prevention and control measures in China, Italy, Iran, South Korea, and Japan was assessed, and the COVID19 epidemic tendency among these countries was compared. Results showed that the effective reproduction number $(R e)$ in Wuhan, China increased almost exponentially, reaching a maximum of 3.98 before a lockdown and rapidly decreased to below 1 due to containment and mitigation strategies of the Chinese government. The $R e$ in Italy declined at a slower pace than that in China after the implementation of prevention and control measures. The $R e$ in Iran showed a certain decline after the establishment of a national epidemic control command, and an evident stationary phase occurred because the best window period for the prevention and control of the epidemic was missed. The epidemic in Japan and South Korea reoccurred several times with the $R e$ fluctuating greatly. The epidemic has hardly rebounded in China due to the implementation of prevention and control strategies and the effective enforcement of policies. Other countries suffering from the epidemic could learn from the Chinese experience in containing COVID-19.
\end{abstract}

Keywords COVID-19; epidemic control comparison; Chinese experience

\section{Introduction}

Coronavirus disease 2019 (COVID-19) is a highly contagious disease spreading globally in early 2020 , with over 200 countries and regions having been affected by April 1, 2020 [1,2]. The World Health Organization (WHO) indicated that COVID-19 could still be contained in countries through containment and mitigation measures $[3,4]$. COVID-19 is caused by a novel coronavirus. The virus can be transmitted from humans to humans through close contact [5,6]. Fever, dyspnea, and dry cough accompanied by typical radiographic features of the lungs are the main clinical symptoms of this disease $[7,8]$. Efforts were made to achieve efficient COVID-19 treatment, whereas effective vaccines and drugs remain in research phases $[9,10]$.

Human contact affects the epidemics of infectious diseases [11]. Generally, infectious, exposed, and

Received March 20, 2020; accepted April 8, 2020

Correspondence: Yankai Xia, yankaixia@njmu.edu.cn recovered individuals are the core factors affecting the epidemic efficiency of infectious diseases [12]. Effective policies can reduce the transmission efficiency of infectious diseases by blocking infectious areas, collecting medical resources to cure patients, and suggesting the public to take personal protective measures (such as wearing masks and washing hands) [13]. Different countries have taken different measures to contain the pandemic.

In China, COVID-19 was controlled by a series of comprehensive measures. On January 23, 2020, the city of Wuhan was locked down. Subsequently, epidemic prevention and control measures were adopted nationwide, including travel restrictions, contact tracing, quarantine, extending the Spring Festival holiday, and improving public health response levels. Huoshenshan Hospital and the innovative Mobile Cabin Hospitals, which started treating patients on February 4 and 5, 2020, respectively, were rapidly built, and medical teams from over 20 provinces rushed to Hubei Province. In particular, on February 12, 2020, Hubei Province started to include clinically diagnosed cases into the confirmed cases 
category to strengthen its quarantine/isolation measures. On February 16, 2020, the New Coronavirus Pneumonia Prevention and Control Plan was further revised to clarify and enhance public health interventions in four key areas: quarantine high-risk individuals, test suspected individuals, treat patients, and receive and cure all patients. During these periods, the resumption of work in most provinces and schools was postponed until the outbreak improved. Consequently, these strategies cut off the source of transmission, minimized the epidemic, and steadily increased the number of cured individuals.

Nevertheless, the COVID-19 epidemic spread rapidly across the world. We focused on the control measurements of Italy, Iran, Japan, and South Korea - four countries that are mainly affected. On January 31, 2020, the government of Italy declared a national emergency with cancellation of round-trip flights from Italy to China before the two sides agreed to resume flights on February 7, 2020. The Basilicata region imposed a 14-day quarantine on individuals from epidemic areas in Northern Italy on February 24, 2020, and school events in Lombard, Veneto, Piemonte, and Emilia-Romagna were cancelled until March 1, 2020. On March 1, 2020, Italy was divided into three regions (red, yellow, and safe), where different control measures were taken, especially in the red and yellow regions. On February 19, 2020, Iran announced two deaths caused by COVID-19 without travel history, marking the beginning of an outbreak. As several suspected cases were found in Qom City, related close contacts were quarantined. On February 20, 2020, Qom City's health department closed schools and proposed to cancel religious activities. By February 24, 2020, over 250 individuals were quarantined, and the National Epidemic Control Command had been established. In Japan, after February 5, 2020, guests on board the "Diamond Princess" were quarantined for 14 days. On February 25, 2020, the government published the basic guidelines for managing sporadic, unexplained cases of infection in many parts of the country. On February 27, 2020, primary and secondary schools across the country suspended courses from March 2, 2020 until the end of spring break. In South Korea, COVID-19's epidemic alert level was raised to the highest on February 23, 2020. On February 26, 2020, three new laws targeting COVID-19 were passed unanimously. On March 2, 2020, South Korea's education ministry announced that all schools across the country would reopen on March 23, 2020. We compared the strategies adopted by these countries and found that the execution of prevention and control strategy was not effective in Japan and South Korea.

Considering the different measures in controlling COVID-19, we compared different COVID-19 epidemic trends in China, Italy, Iran, Japan, and South Korea according to different epidemic control strategies.

\section{Methods}

\section{Data source}

The epidemic statistics in China from January 10 to March 12, 2020 were collected from the National Health Commission of the People's Republic of China. Considering different epidemic outbreak time points in other countries, the epidemic data of Italy (from January 30 to March 12, 2020), Iran (from February 20 to March 12, 2020), Japan (from January 16 to March 12, 2020), and South Korea (from January 20 to March 12, 2020) were acquired from the WHO.

\section{Statistical analysis}

The number of new local cases and new imported cases on day $0-T$ can be determined using the equations $\left\{L_{t}\right\}_{t=0}^{T}$ and $\left\{M_{t}\right\}_{t=0}^{T}$. We assumed that $L_{t}$ and $M_{t}$ follow the Poisson distribution.

Solution $1[14,15]$ :

$$
E\left[L_{t}\right]=R_{0} \sum_{j=0}^{t} p_{j}\left(L_{t-j}+\alpha M_{t-j}\right)
$$

where $E\left[L_{t}\right]$ represents the mean value of the random variable, $p_{j}$ represents the distribution of serial interval (SI), and $\alpha$ quantifies the relative contribution of imported cases to secondary disease transmission. It is assumed that $i \neq j, L_{i}$ and $L_{j}$ are independent. Given the distribution of SI and the observed values of $\left\{L_{t}\right\}_{t=0}^{T}$ and $\left\{M_{t}\right\}_{t=0}^{T}, R_{0}$ was calculated by the maximum likelihood estimation [16].

Solution 2:

We assumed that the number of early local cases corresponds to exponential growth and calculated exponential growth rate $r$ by fitting the number of new local cases $\left\{L_{t}\right\}_{t=0}^{T}$ per day during this stage by Poisson regression [17].

$$
R_{0}=\frac{1}{M(-r)}
$$

where $M$ represents the moment generating function of SI.

$$
E\left[L_{t}\right]=R_{t} \sum_{j=1}^{t} p_{j}\left(L_{t-j}+\alpha M_{t-j}\right)
$$

The effective reproduction number is the average number of patients who can be infected if their symptoms begin at time $t$ [18]. Based on this propagation model, the prior distribution of $R_{t}$ is gamma distribution [19,20]; the analytical form of $R_{t}$ posterior distribution can be obtained under the Bayesian framework. In this study, Re refers to the basic reproduction number at time point $t$. 
Assuming that SI follows the gamma distribution, the mean and standard deviation of SI as estimated by confirmed case information in Shaanxi Province are 5.6 and 3.7 (SI2), respectively. Currently, published research show different estimation results for SI. Previous reports show a mean and standard deviation of 7.5 and 3.4 (SI3) [21] and 4.7 and 2.9 (SI1) [22], respectively. We estimated the corresponding $R_{0}$ and $R e$ for these three SIs.

We assumed that the time of diagnosis for $i$ and $i+1$ confirmed cases in a country is $T_{i}$ and $T_{i+1}$. The interval time of a country is defined as $T=\max _{1 \leqslant i}\left\{T_{i+1}-T_{i}\right\}$ and $a=\arg \max _{1 \leqslant i}\left\{T_{i+1}-T_{i}\right\}$. The stage before $T_{i}$ is defined as stage 1. Stage 2 occurs from $T_{i+1}$ to March 12, 2020. Therefore, the probability that the outbreak of stage 2 was caused by the case of stage 1 is

$$
p_{12}=1-\prod_{j=1}^{a} P\left(\mathrm{SI}<\left(T_{j=1}-T_{j}\right)\right)
$$

The probability of the above countries is low (Table S1). Thus, the outbreak probably started in stage 2 , which is not associated with the cases during stage 1 . Therefore, the calculation of the regeneration number related to these countries was only based on cases in stage 2 . With the exception of the above-mentioned countries, we used the number of new cases in Italian stage 2 (February 22 to March 12, 2020) to calculate the Italian $R_{0}$ and $R e$. The daily number of new cases from January 20 to February 28, 2020 in South Korea was used to calculate $R_{0}$, and the daily number of new cases from January 20 to March 12 , 2020 was used to calculate $R e$. The estimated width of the sliding time window [18] of $R e$ was 3 days. In South Korea, $R_{0}$ and $R e$ are not sensitive to the change of parameter $\alpha$. The SI and Re estimates of three different countries are different. However, the change trend and key time points are similar (such as the time when $R_{0}$ drops below 1 or $R_{0}$ reaches the peak). The $R e$ shown in the main body is the corresponding result of SI (mean: 4.7; SD: 2.9). The $R e$ of Italy remains high until March 12, 2020, indicating that the epidemic is still in a rapid growth stage.

\section{Results}

Before January 23, 2020, the traditional Chinese Spring Festival markedly increased population mobility, leading to an increased risk of COVID-19's spread. During this period, our estimations showed that the $R e$ increased almost exponentially, reaching a maximum of 3.98 on January 21, 2020 before the lockdown of Wuhan. As a result, the number of confirmed cases increased rapidly. However, the number of suspected cases remained at a plateau, indicating that the detection rate was increasing and that contact tracing and quarantine measures were effective. Thus, the $R e$ began to decline. The $R e$ was almost stable around February 5, which fell below 1 after February 9, 2020. More details about the epidemic control strategies in China are shown in Fig. 1.

The prevention and control strategies in Italy, Japan,

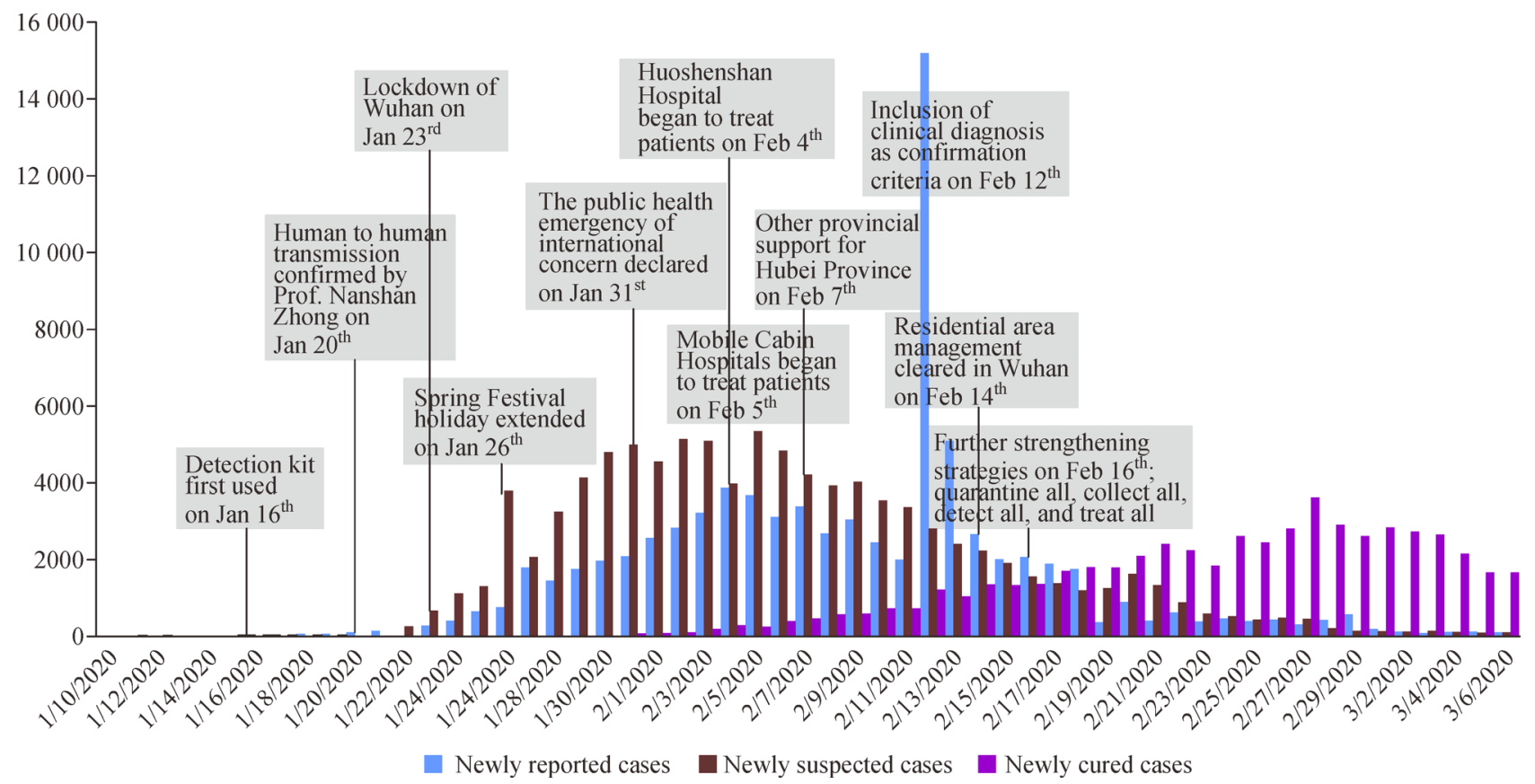

Fig. 1 Time series of the number of newly reported, newly suspected, and newly cured cases with the time points of implementation of enhanced containment and mitigation interventions. 
Iran, and South Korea are listed as a time series in Fig. 2, with newly reported cases. We compared the estimated $R e$ in the five countries. Given the different COVID-19 epidemic onset time in these five countries, we unified the epidemic time series as Day 1 to Day 29 (Table S2). The estimated $R e$ values are shown in Fig. 3 and Table 1.

Table 1 Summary of the effective reproduction number $(R e)$ in the five countries

\begin{tabular}{lll}
\hline \multirow{2}{*}{ Countries } & \multicolumn{2}{c}{ Re } \\
\cline { 2 - 3 } & Day 1 & Day 29 \\
\hline China & 2.80 & 0.51 \\
Italy & 3.47 & 8.01 \\
Iran & 3.90 & 7.90 \\
South Korea & 3.20 & 6.40 \\
Japan & 2.97 & 3.91 \\
\hline
\end{tabular}

\section{Discussion}

The WHO announced on March 12, 2020 that COVID-19 had become a pandemic [3]. High contagiousness and large-scale international traveling have driven COVID-19 to spread rapidly worldwide $[23,24]$. The epidemic in China peaked in early February, 2020 and is currently under control. Much fewer confirmed cases are currently reported compared with the large number of diagnosed cases a month before [25]. However, the global epidemic has worsened in the past two weeks [26,27]. A total number of 16462 confirmed cases was reported in Italy on March 12, 2020, compared with 1694 cases on March 1, 2020. In addition, the mortality due to COVID-19 in Italy was reported to be $4.96 \%$ higher than that in any other countries worldwide. Similarly, the outbreak in South Korea is growing rapidly, with 7869 infected individuals by March 12, 2020. The statistics above indicates that COVID-19 has become a serious global public health emergency [28-30]. To better understand the trend of the epidemic, we estimated $R e$ values in China, Italy, Iran, Japan, and South Korea on the basis of published and reported epidemic statistics.

Since the lockdown of Wuhan, China, the Re has gradually decreased by continuous strengthening of prevention and control measures. Although a slight rebound occurred due to limited medical resources, the situation eased soon after national rescue and the use of the Huoshenshan Hospital and Mobile Cabin Hospitals. The detection rate of suspected cases has been a key factor affecting the epidemic trend, which depends on the implementation of prevention and control strategies and self-isolation measures by communities and individuals $[25,31]$. Given the strict implementation of the prevention and control strategies and the high compliance of individual self-isolation in other provinces in China, the $R e$ rapidly fell below 1 after February 9, 2020. Comprehensive prevention and control measures have achieved initial success, as evidenced by a reduction in the number of new cases. The Re has been less than 1 for a long time since February 10, 2020. Compared with Wuhan after the lockdown, the $R e$ of Italy also decreased but to a smaller extent and rose again in the later period due to limited medical resources. Iran's prevention and control measures are evidently inferior to those of China. Although the $R e$ was reduced to a certain extent, a stable period subsequently appeared (i.e., the optimal control window period was missed). The Re did not decrease until the recent implementation of two nationwide prevention and control strategies. The main features of outbreaks in Japan and South Korea are the inadequate implementation of prevention and control strategies, which has resulted in multiple recurrences of the epidemic and great fluctuation of $R e$. Notably, the progress made in the prevention and control of the epidemic in South Korea gradually showed preliminary effects, with the number of new confirmed cases below 100 for two consecutive days from February 14 to 15,2020 . The government has strong prevention and control efforts and responded quickly [32]. On March 1, 2020 , South Korea announced a state of war. In terms of prevention and control, the government conducted a series of management strategies such as tracing the source of the disease, preventing the spread of the disease, focusing on detection, greatly improving the detection efficiency, and shortening the detection process and cycle. A key step in prevention and control is to identify close contacts of confirmed and suspected cases and prevent the occurrence of unknown second- or third-generation cases. The Korean Centers for Disease Control and Prevention monitors patient exposure through medical facility records, global positioning system, credit card transaction records, and closed-circuit television information. Quarantine and isolation were strictly applied to control the epidemic. In addition, the public awareness of the epidemic has been raised [32]. The government's cooperation in the prevention and control of the epidemic has greatly improved, which is optimistic for the control against COVID-19.

According to the comparison of $R e$ between countries, the enforcement of Chinese policies is remarkably more effective than that of several other countries worldwide. Since the implementation of the prevention and control strategy, the epidemic has hardly rebounded in China. However, outbreaks in South Korea and Japan have repeatedly occurred, and the $R e$ has not fallen below 1 , indicating that the epidemic situation has not been effectively controlled and that the number of cumulative cases will continue to increase (Fig. 3). Furthermore, given the limited effectiveness of the early prevention and control strategies, the situation in Iran is still not optimistic. 

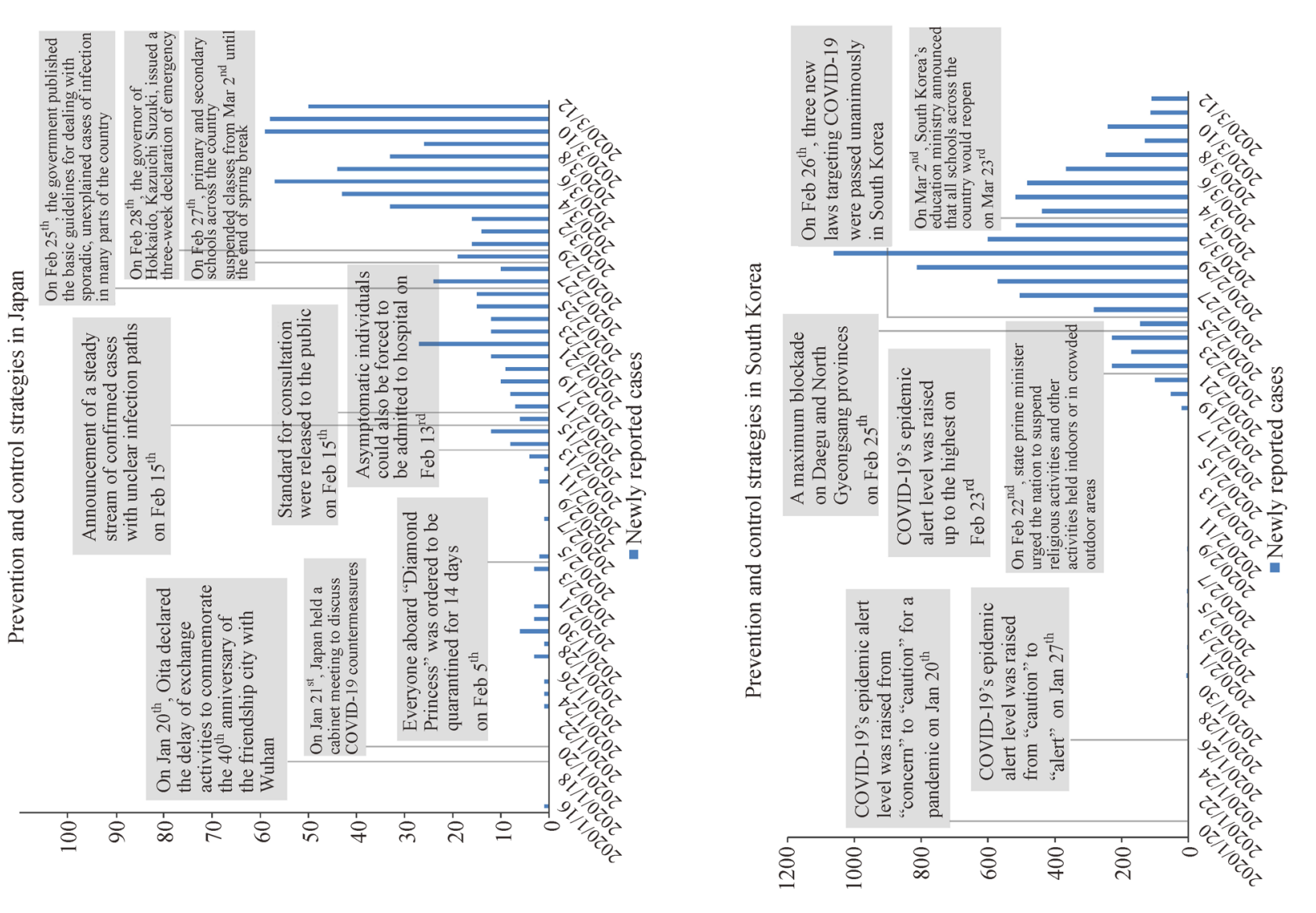


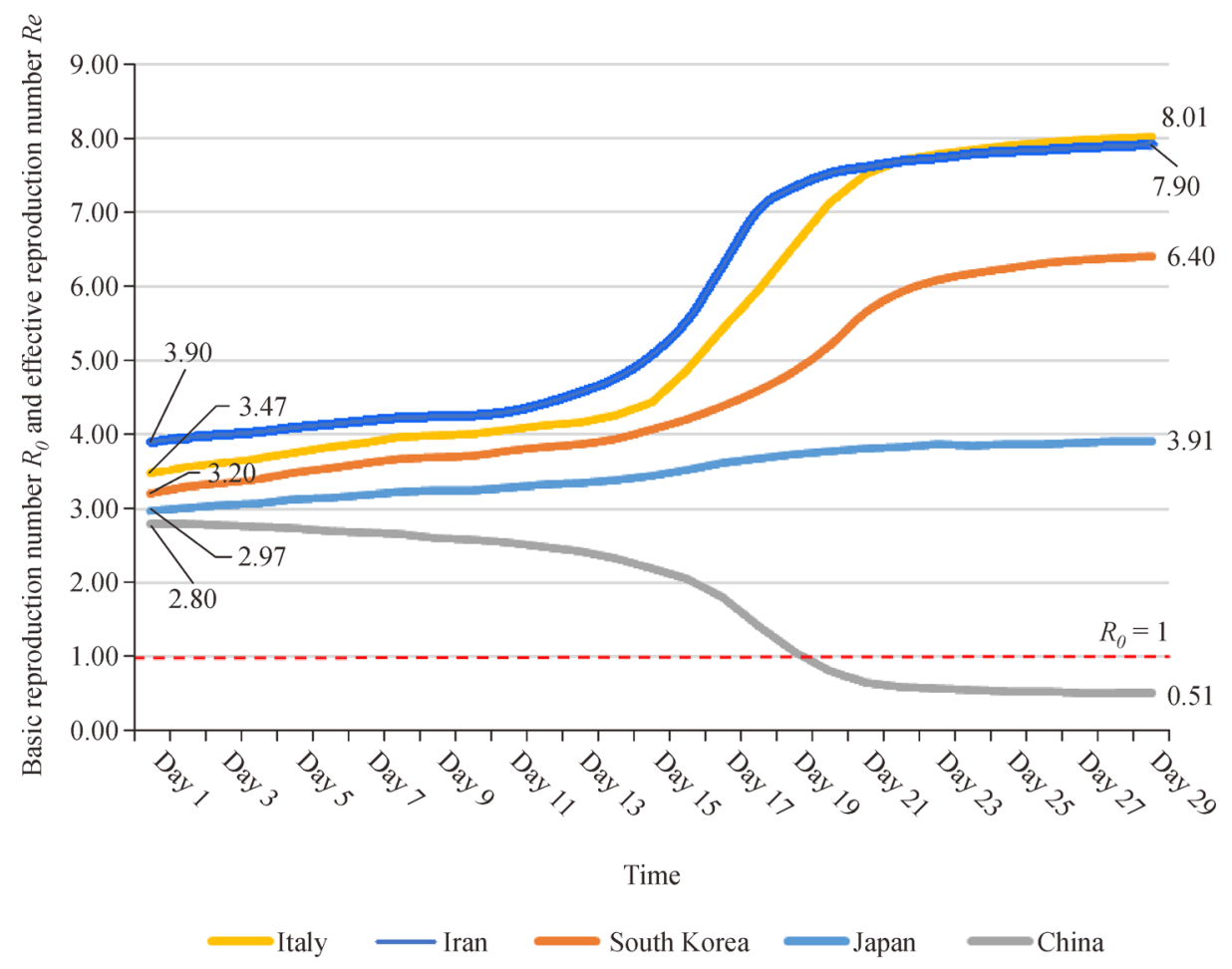

Fig. 3 Comparison of the trends of the effective reproduction number of COVID-19 among the five countries.

The trend of the epidemic in Iran and Italy depends on the strength and execution of the later prevention and control strategies and public compliance.

Despite the findings, this study has several limitations. Considering the change of parameters due to practical situations, such as continuous control measures, the model should update dynamically according to the updated epidemic statistics. Qualitative description was applied to explain the rapid decline of $R e$ in China without considering other confounding factors. Further studies should focus more on the causal relationship between control measures and the COVID-19 epidemic.

\section{Acknowledgements}

The study was supported in part by the National Natural Science Foundation of China (Nos. 81673275 and 91846302), the National S\&T Major Project Foundation of China (Nos. 2018ZX10715002004 and 2018ZX10713001-001), and the Priority Academic Program Development of Jiangsu Higher Education Institutions (PAPD).

\section{Compliance with ethics guidelines}

Zhihang Peng, Wenyu Song, Zhongxing Ding, Quanquan Guan, Xu Yang, Qiaoqiao Xu, Xu Wang, and Yankai Xia declare that they have no actual or potential competing financial interests. This article does not include studies involving human or animal subjects.
Electronic Supplementary Material Supplementary material is available in the online version of this article at https://doi.org/ $10.1007 / \mathrm{s} 11684-020-0788-3$ and is accessible for authorized users.

\section{References}

1. Zhou P, Yang XL, Wang XG, Hu B, Zhang L, Zhang W, Si HR, Zhu Y, Li B, Huang CL, Chen HD, Chen J, Luo Y, Guo H, Jiang RD, Liu MQ, Chen Y, Shen XR, Wang X, Zheng XS, Zhao K, Chen QJ, Deng F, Liu LL, Yan B, Zhan FX, Wang YY, Xiao GF, Shi ZL. A pneumonia outbreak associated with a new coronavirus of probable bat origin. Nature 2020; 579(7798): 270-273

2. Carvalho T. COVID-19 research in brief: 28 March to 3 April, 2020. Nat Med 2020. [Epub ahead of print] doi: 10.1038/d41591-02000008-y

3. Mahase E. COVID-19: WHO declares pandemic because of "alarming levels" of spread, severity, and inaction. BMJ 2020; 368: $\mathrm{m} 1036$

4. Shah SGS, Farrow A. A commentary on "World Health Organization declares global emergency: a review of the 2019 novel coronavirus (COVID-19)". Int J Surg 2020; 76: 128-129

5. Guo YR, Cao QD, Hong ZS, Tan YY, Chen SD, Jin HJ, Tan KS, Wang DY, Yan Y. The origin, transmission and clinical therapies on coronavirus disease 2019 (COVID-19) outbreak - an update on the status. Mil Med Res 2020; 7(1): 11

6. Peng Y, Zhou YH. Is novel coronavirus disease (COVID-19) transmitted through conjunctiva? J Med Virol 2020; jmv.25753

7. Rodriguez-Morales AJ, Cardona-Ospina JA, Gutiérrez-Ocampo E, 
Villamizar-Peña R, Holguin-Rivera Y, Escalera-Antezana JP, Alvarado-Arnez LE, Bonilla-Aldana DK, Franco-Paredes C, Henao-Martinez AF, Paniz-Mondolfi A, Lagos-Grisales GJ, Ramírez-Vallejo E, Suárez JA, Zambrano LI, Villamil-Gómez WE, Balbin-Ramon GJ, Rabaan AA, Harapan H, Dhama K, Nishiura H, Kataoka H, Ahmad T, Sah R; Latin American Network of Coronavirus Disease 2019-COVID-19 Research (LANCOVID19). Clinical, laboratory and imaging features of COVID-19: a systematic review and meta-analysis. Travel Med Infect Dis 2020. [Epub ahead of print] doi: 10.1016/j.tmaid.2020.101623

8. Cheng Z, Lu Y, Cao Q, Qin L, Pan Z, Yan F, Yang W. Clinical features and chest CT manifestations of coronavirus disease 2019 (COVID-19) in a single-center study in Shanghai, China. AJR Am J Roentgenol 2020. [Epub ahead of print] doi: 10.2214/AJR.20.22959

9. Jiang S. Don't rush to deploy COVID-19 vaccines and drugs without sufficient safety guarantees. Nature 2020; 579(7799): 321

10. Cortegiani A, Ingoglia G, Ippolito M, Giarratano A, Einav S. A systematic review on the efficacy and safety of chloroquine for the treatment of COVID-19. J Crit Care 2020. [Epub ahead of print] doi: 10.1016/j.jcrc.2020.03.005

11. Ajelli M, Poletti P, Melegaro A, Merler S. The role of different social contexts in shaping influenza transmission during the 2009 pandemic. Sci Rep 2015; 4: 7218

12. Etbaigha F, R Willms A, Poljak Z. An SEIR model of influenza A virus infection and reinfection within a farrow-to-finish swine farm. PLoS One 2018; 13(9): e0202493

13. Park JE, Jung S, Kim A, Park JE. MERS transmission and risk factors: a systematic review. BMC Public Health 2018; 18(1): 574

14. Nishiura H, Roberts MG. Estimation of the reproduction number for 2009 pandemic influenza $\mathrm{A}(\mathrm{H} 1 \mathrm{~N} 1)$ in the presence of imported cases. Euro Surveill 2010; 15(29): 19622

15. Shim E, Tariq A, Choi W, Lee Y, Chowell G. Transmission potential and severity of COVID-19 in South Korea. Int J Infect Dis 2020; 93 : 339-344

16. White LF, Wallinga J, Finelli L, Reed C, Riley S, Lipsitch M, Pagano M. Estimation of the reproductive number and the serial interval in early phase of the 2009 influenza $\mathrm{A} / \mathrm{H} 1 \mathrm{~N} 1$ pandemic in the USA. Influenza Other Respir Viruses 2009; 3(6): 267-276

17. Wallinga J, Lipsitch $M$. How generation intervals shape the relationship between growth rates and reproductive numbers. Proc Biol Sci 2007; 274(1609): 599-604

18. Cori A, Ferguson NM, Fraser C, Cauchemez S. A new framework and software to estimate time-varying reproduction numbers during epidemics. Am J Epidemiol 2013; 178(9): 1505-1512

19. Nikbakht R, Baneshi MR, Bahrampour A. Estimation of the basic reproduction number and vaccination coverage of influenza in the United States (2017-18). J Res Health Sci 2018; 18(4): e00427

20. Wang X, Shi Y, Feng Z, Cui J. Evaluations of interventions using mathematical models with exponential and non-exponential distributions for disease stages: the case of Ebola. Bull Math Biol 2017; 79(9): 2149-2173

21. Li Q, Guan X, Wu P, Wang X, Zhou L, Tong Y, Ren R, Leung KSM, Lau EHY, Wong JY, Xing X, Xiang N, Wu Y, Li C, Chen Q, Li D, Liu T, Zhao J, Liu M, Tu W, Chen C, Jin L, Yang R, Wang Q, Zhou S, Wang R, Liu H, Luo Y, Liu Y, Shao G, Li H, Tao Z, Yang Y, Deng Z, Liu B, Ma Z, Zhang Y, Shi G, Lam TTY, Wu JT, Gao GF, Cowling BJ, Yang B, Leung GM, Feng Z. Early transmission dynamics in Wuhan, China, of novel coronavirus-infected pneumonia. N Engl J Med 2020; 382(13): 1199-1207

22. Nishiura H, Linton NM, Akhmetzhanov AR. Serial interval of novel coronavirus (COVID-19) infections. Int J Infect Dis 2020; 93: 284286

23. Zhong P, Guo S, Chen T. Correlation between travellers departing from Wuhan before the Spring Festival and subsequent spread of COVID-19 to all provinces in China. J Travel Med 2020; 14(2): 113-116

24. Hunter P. The spread of the COVID-19 coronavirus: health agencies worldwide prepare for the seemingly inevitability of the COVID-19 coronavirus becoming endemic. EMBO Rep 2020; 21(4): e50334

25. Zhou G, Chen S, Chen Z. Back to the spring of 2020: facts and hope of COVID-19 outbreak. Front Med 2020; 14(2): 113-116

26. Remuzzi A, Remuzzi G. COVID-19 and Italy: what next? Lancet 2020. [Epub ahead of print] doi: 10.1016/S0140-6736(20)30627-9

27. Mahase E. COVID-19: outbreak could last until spring 2021 and see 7.9 million hospitalised in the UK. BMJ 2020; 368: $\mathrm{m} 1071$

28. Livingston E, Bucher K. Coronavirus disease 2019 (COVID-19) in Italy. JAMA 2020. [Epub ahead of print] doi: 10.1001/ jama.2020.4344

29. Green MS. Did the hesitancy in declaring COVID-19 a pandemic reflect a need to redefine the term? Lancet 2020; 395(10229): 10341035

30. The Lancet Infectious Diseases. COVID-19, a pandemic or not? Lancet Infect Dis 2020; 20(4): 383

31. Gong F, Xiong Y, Xiao J, Lin L, Liu X, Wang D, Li X. China's local governments are combating COVID-19 with unprecedented responses - from a Wenzhou governance perspective. Front Med 2020; 14(2): 220-224

32. Korean Society of Infectious Diseases; Korean Society of Pediatric Infectious Diseases; Korean Society of Epidemiology; Korean Society for Antimicrobial Therapy; Korean Society for Healthcareassociated Infection Control and Prevention; Korea Centers for Disease Control and Prevention. Report on the epidemiological features of coronavirus disease 2019 (COVID-19) outbreak in the Republic of Korea from January 19 to March 2, 2020. J Korean Med Sci 2020; 35(10): e112 